\title{
Study on stability and in vitro digestion of camellia oil nanoemulsion system
}

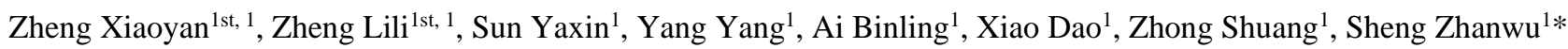 \\ ${ }^{1}$ Haikou Experimental Station, Chinese Academy of Tropical Agricultural Sciences, Haikou, China
}

\begin{abstract}
The soy protein isolate (SPI) was combined with tea saponin as an emulsifier to prepare camellia oil nanoemulsion, and the stability of camellia oil nanoemulsion was compared with that of soybean protein isolate or tea saponin as emulsifier.The effects of different $\mathrm{pH}$, ionic strength, heating temperature and storage time on the average particle size, $\xi$-potential and microstructure of camellia oil nanoemulsion prepared by three emulsifiers were studied. The results showed that the nanoemulsions prepared by combining natural emulsifiers (SPI-TS) in the $\mathrm{pH}$ range of 5-9 were stable and remained stable in the range of $0-300 \mathrm{~mm} \mathrm{NaCl}$ concentration, but had poor tolerance to high salt environment. After heating at different temperatures $\left(30{ }^{\circ} \mathrm{C}-90{ }^{\circ} \mathrm{C}\right.$ ) for $30 \mathrm{~min}$, the average particle size, $\xi$-potential and microstructure of the three emulsions did not change significantly, showing good heating stability. At different storage temperatures $\left(4,25,50^{\circ} \mathrm{C}\right)$ SPI-TS and TS emulsion could exist stably about four weeks, and had good storage stability. In addition, we performed in vitro simulated gastrointestinal digestion studies on the digestive properties of camellia oil nanoemulsions. The results showed that the particle size, the $\xi$-potential of the nanoemulsion changes depend on the type of emulsifier during digestion. The release rate of free fat acids (FFAs) of nanoemulsions after gastrointestinal digestion were all higher than that of the control group. The results showed that the nanoemulsion delivery system could effectively improve the digestion of camellia oil, It was important to improve the bioavailability of camellia oil.
\end{abstract}

\section{Introduction}

Camellia Lutchuensis plants belong to the Camellia family (Theaceae Mirbel) and are composed of more than 325 species ${ }^{[1]}$. Obtained from camellia seeds, camellia oil is used as a cooking vegetable oil in traditional Chinese cuisine. Studies have shown that the nutritional value of camellia oil is extremely high, with unsaturated fatty acids as high as $80 \%$, among which oleic acid content is as high as $74 \%-85 \%{ }^{[2]}$. Because of its similar fatty acid composition with olive oil, camellia oil is known as oriental olive oil and is becoming more and more popular among consumers in East Asia ${ }^{[3,4]}$. Camellia oil has benefits for human health, such as lowering blood pressure and atherosclerosis and scavenging free radicals ${ }^{[5,6]}$. Camellia oil is also rich in unsaponifiables that are beneficial to health, such as sterols, fatty alcohols, tocopherols, triterpene alcohols and squalene ${ }^{[7,8,9]}$. Camellia oil has great potential as functional food and health food. However, because camellia oil is rich in unsaturated fatty acids, it is very susceptible to spoilage due to heat, light, oxygen and other factors, which limits the application range of camellia oil.

For substances that are easily oxidized, fat-soluble, and difficult to absorb (such as lipids, vitamins, etc.), Nanoemulsion is an effective encapsulated delivery system ${ }^{[10]}$. Nanoemulsion is a system in which two immiscible solutions (water and oil) exist stably in the same phase through the interfacial film formed by surfactant molecules ${ }^{[11]}$. A large number of studies have proved that nanoemulsion can carry various functional ingredients (such as carotenoids, lipids, vitamins, etc.) and improve its stability and bioavailability [12,13]. Emulsion is a thermodynamically unstable system that can be decomposed by various means, including emulsification, flocculation, coalescence, Ostwald ripening and phase separation ${ }^{[14]}$. Choosing a suitable emulsifier is very important for the successful preparation of emulsions in commercial applications, because the particle size of the emulsion and its particle size distribution range will significantly affect the appearance, texture, rheology and stability of the food ${ }^{[15]}$ Studies have also shown that the digestive properties of nanoemulsions are affected by the types of emulsifiers. At the same time, physical and biochemical conditions in different parts of the human digestive system, such as shear force, $\mathrm{pH}$, ionic strength, digestive enzymes, etc., all affect the digestive properties of nanoemulsions ${ }^{[16-19]}$. In response to consumer demand for healthier, more natural, and more environmentally friendly food, the food industry is trying to replace synthetic emulsifiers with natural emulsifiers. In recent years, scholars from various countries have carried out a large number of

\footnotetext{
* Corresponding author: shengzhanwu100@163.com
} 
natural emulsifiers (including polysaccharides, proteins, phospholipids and saponins, etc.) to prepare emulsion research [20]

Soy Protein Isolate (SPI) has functional properties such as amphiphilicity and emulsification. It is a cheap and high-quality plant protein. Soy protein isolate can be used to prepare oil-in-water nanoemulsion ${ }^{[21]}$. However, the variability of protein under different conditions (such as $\mathrm{pH}$, ion concentration) leads to poor environmental stability of nanoemulsions prepared as emulsifiers alone. Therefore, this paper studies the combination of soy protein isolate and other emulsifiers to improve the emulsification of protein ${ }^{[22,23]}$. Tea Saponin (TS) is a natural, biodegradable, environmentally friendly emulsifier, mainly derived from camellia seed cake after oil extraction, which can effectively stabilize the emulsion and can be used for commercial products ${ }^{[24,25]}$. In this study, SPI-TS composite emulsifier and camellia oil were used to prepare camellia oil nanoemulsions through high-pressure micro jet homogenization. The stability and digestibility of the camellia oil nanoemulsions under different conditions (such as $\mathrm{pH}$, ionic strength, and temperature) were investigated. The camellia oil nanoemulsion prepared by TS emulsifier was used to compare and analyze the bioavailability of camellia oil in different systems. The aim is to establish a natural green, low-cost, high-stability nanoemulsification system, improve the stability and bioavailability of camellia oil, and broaden the application of camellia oil in the fields of food, chemicals, medicine, etc.

\section{Materials and methods}

\subsection{Materials and reagents}

Tea saponin with $65 \%$ purity is produced by Shanghai Yuanye Biological Co., Ltd.; Soy protein isolate with 98\% purity is produced by Henan Wanbang Industrial Co., Ltd.; Camellia oil is produced by Hainan Xinmeite Technology Co., Ltd.; Disodium hydrogen phosphate, sodium dihydrogen phosphate, sodium chloride and hydroxide are produced by Xilong Chemical Co., Ltd.; Other reagents are of analytical grade.

\subsection{Instruments and equipment}

C25 high-speed shearing dispersion homogenizing emulsifier from Shanghai Hengchuan Machinery Equipment Co., Ltd.; AMH-3 high pressure micro-jet nano homogenizer from ATS Engineering Limited, Canada; Nano-ZS90 particle size analyzer from Malvern, UK; S210 laboratory pH meter from Shanghai MettlerToledo Instrument Equipment Co., Ltd.; AL-104 precision electronic balance from Shanghai MettlerToledo Instrument Equipment Co., Ltd.; FV1000 laser confocal scanning microscope from Olympus Japan; DK-S26 constant temperature water bath from Shanghai Jing Hong Experimental Equipment Co., Ltd.; DHP9162 electric heating constant temperature incubator from Shanghai Yiheng Scientific Instrument Co., Ltd.;
SHA-CA digital display water bath constant temperature oscillator from Changzhou Putian Instrument Manufacturing Co., Ltd.

\subsection{Experimental method}

\subsubsection{Preparation of soy protein isolate-tea saponin camellia oil nanoemulsion}

Dissolve soy protein isolate and tea saponin (mass ratio $1: 2)$ in phosphate buffer solution $(\mathrm{pH} 7.0,0.04 \mathrm{~mol} / \mathrm{L})$, stir continuously for $2 \mathrm{~h}$ at room temperature, and place in a refrigerator at $4^{\circ} \mathrm{C}$ for later use as the water phase. The camellia oil with a mass fraction of $10 \%$ is added to the $3 \%$ emulsifier-containing water phase, homogenized with a high-speed disperser at 10,000 r/min for $2 \mathrm{~min}$ to prepare a coarse emulsion. The coarse emulsion is further homogenized and emulsified for 6 times by a high-pressure micro-jet homogenizer at $100 \mathrm{Mpa}$ to obtain SPI-TS camellia oil nanoemulsion. With reference to the above method, TS camellia oil nanoemulsion and SPI camellia oil nanoemulsion are prepared under the same conditions as a control.

\subsubsection{Study on the stability of camellia oil nanoemulsion}

The stability of SPI-TS camellia oil nanoemulsion, SPI camellia oil nanoemulsion and TS camellia oil nanoemulsion under different conditions $(\mathrm{pH}$, ionic strength, preparation temperature, storage process) is compared and analyzed.

\subsubsection{1 pH stability of camellia oil nanoemulsion}

Put $10 \mathrm{~mL}$ of the three newly prepared camellia oil nanoemulsions into different glass beakers, and adjust the $\mathrm{pH}$ value to $3.0,4.0,5.0,6.0,7.0,8.0,9.0$ with $0.1 \mathrm{M}$ $\mathrm{HCl}$ or $0.1 \mathrm{M} \mathrm{NaOH}$ solution, respectively. Then transfer the sample to a glass test tube and store it at $25^{\circ} \mathrm{C}$ for later use.

\subsubsection{Influence of $\mathrm{NaCl}$ concentration on the stability of camellia oil nanoemulsion}

Transfer $10 \mathrm{~mL}$ of the three newly prepared camellia oil nanoemulsions ( $\mathrm{pH} 7.0$ ) to a glass beaker, add $10 \mathrm{~mL}$ salt solutions of different concentrations to make the final concentration of $\mathrm{NaCl} 0 \mathrm{mM}, 100 \mathrm{mM}, 200 \mathrm{mM}$, $300 \mathrm{mM}, 400 \mathrm{mM}, 500 \mathrm{mM}$, and then transfer the samples to store in a glass test tube at $25^{\circ} \mathrm{C}$ for later use.

\subsubsection{Influence of temperature on the stability of camellia oil nanoemulsion}

Place the three newly prepared camellia oil nanoemulsions $(\mathrm{pH} 7.0)$ in a water bath at a temperature of $30^{\circ} \mathrm{C}, 40^{\circ} \mathrm{C}, 50^{\circ} \mathrm{C}, 60^{\circ} \mathrm{C}, 70^{\circ} \mathrm{C}, 80^{\circ} \mathrm{C}$, and $90^{\circ} \mathrm{C}$ for 30 
minutes, and then cool the sample to room temperature, store at $25^{\circ} \mathrm{C}$ for later use.

\subsubsection{Influence of storage temperature on the stability of camellia oil nanoemulsion}

Place $30 \mathrm{~mL}$ of the newly prepared three camellia oil nanoemulsions $(\mathrm{pH} 7.0)$ in a temperature environment of $4{ }^{\circ} \mathrm{C}, 25{ }^{\circ} \mathrm{C}$, and $50{ }^{\circ} \mathrm{C}$ for 28 days, measure the average particle size, PDI and $\zeta$ - potential of the emulsion every 7 days, and then observe the microscopic morphology of the nanoemulsion.

\subsubsection{Determination of average particle size, PDI and $\zeta$-potential of camellia oil nanoemulsion}

Zetasizer Nano-ZS90 light scattering particle size analyzer is used to measure the average particle size, PDI and $\zeta$-potential of the camellia oil nanoemulsion. The refractive index of the camellia oil droplets is set to 1.45 , and the refractive index of the aqueous solution is set to 1.33. In order to reduce the multiple light scattering effect, the nanoemulsion is diluted 100 times with $\mathrm{pH} 7.0,0.04 \mathrm{~mol} / \mathrm{L}$ phosphate buffer before analysis.

\subsubsection{In vitro digestion of camellia oil nanoemulsion}

The experimental method of WOOSTER et al. ${ }^{[26]}$ is referred and we modify on its basis to construct a gastrointestinal tract model to simulate the digestion of gastric juice and intestinal juice. During the entire experiment, the emulsion digestion sample was placed on a constant temperature shaking shaker at $37^{\circ} \mathrm{C}$ and $200 \mathrm{r} / \mathrm{min}$ for 3 hours.

\subsubsection{Simulated gastric juice digestion}

Weigh $2.000 \mathrm{~g}$ of $\mathrm{NaCl}$ into $7 \mathrm{~mL}$ of $0.1 \mathrm{M} \mathrm{HCl}$, then add deionized water to make the volume to $1 \mathrm{~L}$, and use $1 \mathrm{~mol} / \mathrm{L} \mathrm{HCl}$ to adjust the $\mathrm{pH}$ to 2.0 . Take $30 \mathrm{~mL}$ of the above-prepared solution and add $0.96 \mathrm{~g}$ of pepsin at a concentration of $32 \mathrm{mg} / \mathrm{mL}$ to prepare simulated gastric juice (SGF). Take $30 \mathrm{~mL}$ of nanoemulsion and $30 \mathrm{~mL}$ of SGF and mix well, and adjust the $\mathrm{pH}$ to 2.0 with $0.1 \mathrm{M}$ $\mathrm{HCl}$. After reacting in a shaker at $37^{\circ} \mathrm{C}$ and $200 \mathrm{r} / \mathrm{min}$ for 1 hour, the $\mathrm{pH}$ of the system is adjusted to 7.0 with $\mathrm{NaOH}$ to terminate the enzymatic reaction.

\subsubsection{Simulated digestion of intestinal juice}

Dissolve $10 \mathrm{mg} / \mathrm{mL}$ pancreatin and $12 \mathrm{mg} / \mathrm{mL}$ bile salt in $100 \mathrm{~mL}$ (0.04 M, pH 7.0) phosphate buffer solution, and then adjust the $\mathrm{pH}$ to 7.0 with $0.1 \mathrm{M} \mathrm{NaOH}$ to prepare simulated intestinal fluid (SIF). Take $30 \mathrm{~mL}$ of the emulsion digested by the simulated gastric juice and 30 $\mathrm{mL}$ of SIF and mix them uniformly, adjust the $\mathrm{pH}$ to 7.0 with $\mathrm{NaOH}$, and react for 2 hours in a shaker at $37^{\circ} \mathrm{C}$ and $200 \mathrm{r} / \mathrm{min}$. During the simulated intestinal digestion stage, $\mathrm{NaOH}$ is continuously used to maintain the $\mathrm{pH}$ of the system at 7.0.

\subsubsection{Free fatty acid (FFA) release}

In the process of simulating digestion of intestinal juice, camellia oil is hydrolyzed into free fatty acids under the action of pancreatic lipase and bile salt, and $\mathrm{NaOH}$ consumption in different time intervals $(5,10,20,30,60$, $90,120 \mathrm{~min}$ ) is recorded. According to the consumption of $\mathrm{NaOH}$, the amount of free fatty acids in the system is calculated. Assuming that one molecule of triglyceride produces one molecule of monoglyceride and two molecules of free fatty acid when it is completely digested, the free fatty acid release rate $\mathrm{Y}$ is calculated according to the volume of $\mathrm{NaOH}$ consumed continuously, see formula ${ }^{[27]}$ :

$$
\mathrm{Y}=\frac{\mathrm{V}_{\mathrm{NaOH}} \times \mathrm{m}_{\mathrm{NaOH}} \times \mathrm{M}_{\text {lipid }}}{2 \times \mathrm{m}_{\text {lipid }}} \times 100 \%
$$

Where, $\mathrm{Y}$ is the release rate of free fatty acids; $\mathrm{VNaOH}$ is the volume of $\mathrm{NaOH}$ solution consumed $(\mathrm{mL})$; $\mathrm{mNaOH}$ is the concentration of $\mathrm{NaOH}$ solution at titration $(\mathrm{mol} / \mathrm{L})$; Mlipid is the average molecular weight of camellia oil $(\mathrm{g} / \mathrm{mol})$; Mlipid is the total mass of camellia oil in the emulsion $(\mathrm{g})$.

\subsubsection{Microscopic morphology of camellia oil nanoemulsion}

The confocal laser scanning microscope (CLSM, FV1000, Olympus, Japan) is used to characterize the microstructure of the emulsion at room temperature. According to the method in the literature ${ }^{[28]}$, with a slight modification, before the analysis, $1 \mathrm{~mL}$ of the emulsion is mixed with $0.1 \mathrm{~mL}$ of Nile Red solution (1 $\mathrm{mg} / \mathrm{mL}$ dissolved in ethanol) to stain the oil. The excitation and emission wavelengths of Nile Red are 543 $\mathrm{nm}$ and $605 \mathrm{~nm}$, respectively. Transfer the emulsion sample $(6 \mu \mathrm{L})$ to a microscope slide and cover it with a glass cover glass. Use a $40 \times$ objective lens to capture and analyze the CLSM microscopic image through FV10-ASW3.1 software.

\subsection{Data processing and analysis}

The data are measured in parallel for 3 times and the average value is taken. SPSS Statistics 22 is used to analyze the significance of the difference by ANOVA, and $\mathrm{p}<0.05$ is considered as a significant difference, which is plotted with Origin8.5.

\section{Results and analysis}

\subsection{Study on the stability of camellia oil nanoemulsion}

\subsection{1 pH stability}


Figure 1 shows the effect of different $\mathrm{pH}$ values on the average particle size (a) and $\zeta$-potential (b) of camellia oil nanoemulsions prepared by different emulsifiers. It can be seen from Figure 1a that the average particle size of SPI-TS camellia oil nanoemulsion in the $\mathrm{pH}$ range of 5-9 remains stable, and is basically not affected by changes in $\mathrm{pH}$ value. However, in a strong acid environment, that is, when the $\mathrm{pH}$ is 3 and 4, the average particle size of SPI-TS nanoemulsion has increased significantly $(\mathrm{p}<0.05)$, from about $200 \mathrm{~nm}$ to more than $1000 \mathrm{~nm}$. On the other hand, the average particle size did not change significantly under alkaline conditions, indicating that the peracid environment $(\mathrm{pH}<4)$ is not conducive to the stability of SPI-TS camellia oil nanoemulsion, but it can exist stably in alkaline environment. At the same time, it can be seen from Figure 1a that the SPI emulsion is extremely unstable in acidic environment, and the average particle size is significantly higher than that under alkaline conditions $(\mathrm{p}<0.05)$, especially in the range of $\mathrm{pH} 3-5$, which may be because this $\mathrm{pH}$ range is at the isoelectric point of soy protein isolate, the electrostatic repulsion between droplets is reduced ${ }^{[29]}$. It can also be seen from Figure 1a that the average particle size of TS nanoemulsion at $\mathrm{pH} 3$ is higher, but it is relatively stable under other $\mathrm{pH}$ conditions.

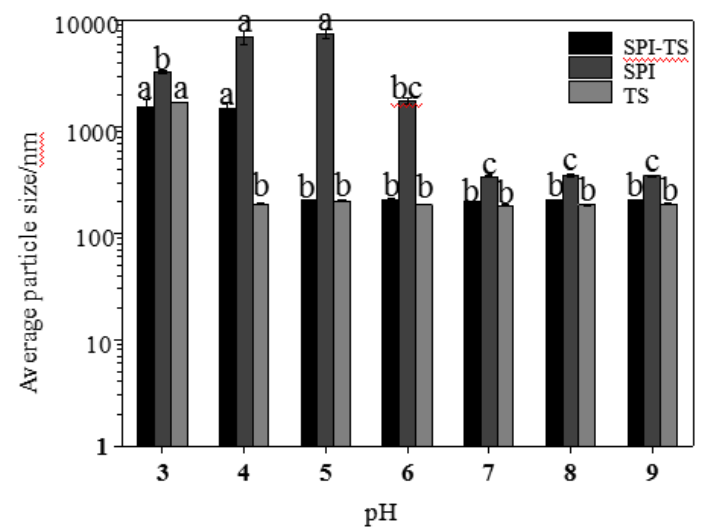

(a)

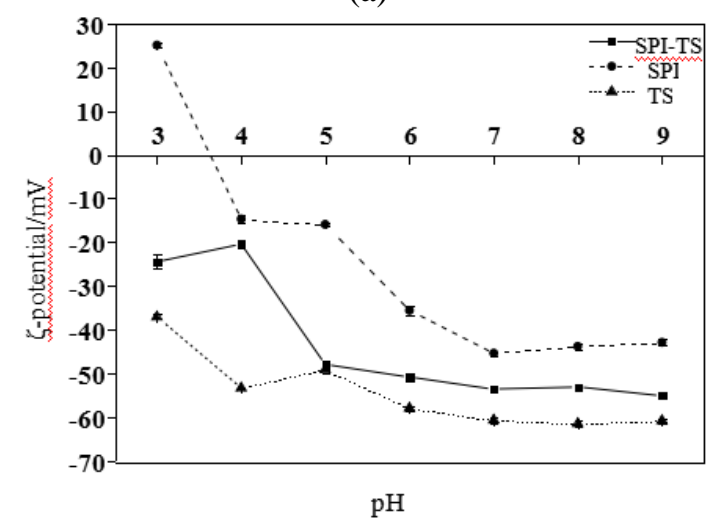

(b)

Fig.1 Effects of different $\mathrm{pH}$ values on (a) average particle size and (b) $\xi$-potential of three types camellia oil nanoemulsion

Note: The lowercase letters represent the significant difference of the same emulsion, $\mathrm{p}<0.05$ is the significant difference, the same below.
Figure $1 b$ is the graph of the $\zeta$-potential changes of three camellia oil nanoemulsions. It can be seen that when the $\mathrm{pH}$ of the SPI-TS and TS nanoemulsions is greater than 6 , the $\zeta$-potential basically remains unchanged, and the emulsion exhibits a higher absolute value of the $\zeta$-potential. Studies have shown that the negative surface potential on the emulsion droplets is caused by the carboxylic acid groups on the saponins molecules adsorbed on the interface, and the greater the absolute value of the $\zeta$ - potential, the more stable the emulsion is ${ }^{[30,31]}$. At $\mathrm{pH}<6$, the absolute value of the $\zeta$ potential of both becomes smaller, indicating that acidic conditions weaken the $\zeta$-potential intensity of the emulsion, and the decrease of the $\zeta$-potential is not conducive to the stability of the emulsion. The $\zeta$ potential of the SPI nanoemulsion becomes positive at $\mathrm{pH}=3$, which may be because the low $\mathrm{pH}$ denatures the protein and reduces the electrostatic repulsion between the droplets [32]. In summary, the SPI-TS and TS nanoemulsions are more resistant to environmental $\mathrm{pH}$ changes.

Figure 2 is the laser confocal scanning microscope image of three nanoemulsions at different $\mathrm{pH}$. It can be seen from the figure that the camellia oil in the emulsion is severely aggregated under acidic conditions in the SPI stable emulsion, and small oil droplets are formed into large oil droplets, consistent with the results shown in Figure 1a. The increase in the average particle size of the emulsion may be due to the denaturation of the protein in this $\mathrm{pH}$ range, and the emulsifier cannot form a tight interfacial film on the surface of the droplet or the interfacial film cannot exist stably on the surface of the droplet, thereby reducing the electrostatic repulsion between the droplets, which gather into large droplets. In the emulsion prepared by the composite emulsifier and the emulsion prepared by TS, the aggregation of large oil droplets is only observed at $\mathrm{pH}=3$, and only a small amount of aggregation is observed. It can be seen that both SPI-TS emulsion and TS emulsion exhibit better $\mathrm{pH}$ environmental tolerance than SPI emulsion when the $\mathrm{pH}$ is greater than 3 .

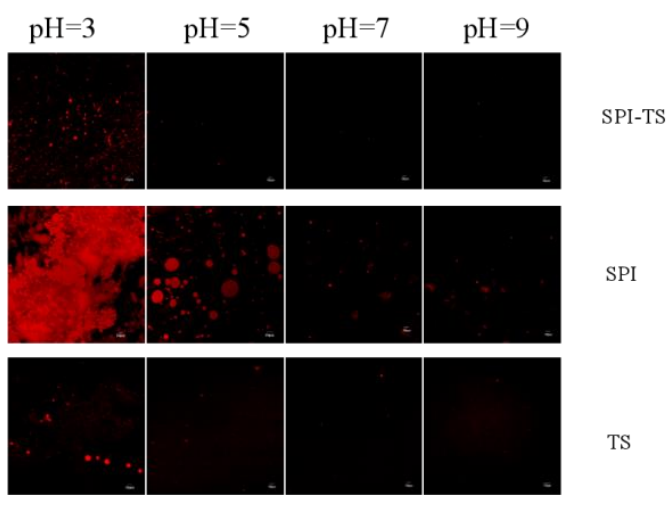

Fig.2 CLSM diagram of composite nanoemulsion, SPI nanoemulsion and TS nanoemulsion at different $\mathrm{pH}$

\subsubsection{Salt ion concentration stability}


Figure 3 shows the effect of different salt ion concentrations on the average particle size and $\zeta$ potential of camellia oil nanoemulsions prepared with different emulsifiers. Salt is a common food additive and exists in the human gastrointestinal tract, which may affect the function of the emulsion carrier system during the preparation of products or after ingestion. Therefore, it is particularly important to study the effect of salt concentration on the nanoemulsion carrier system. From Figure 3a, it can be seen that the average particle size of SPI-TS nanoemulsion does not change significantly in the range of $0-300 \mathrm{mM} \mathrm{NaCl}$ concentration, but it increases with the increase of salt ion concentration when the salt ion concentration is greater than $400 \mathrm{mM}$. This may be because the high concentration of $\mathrm{NaCl}$ decreases the electrostatic repulsion ${ }^{[28]}$ between oil droplets and promotes droplet aggregation, but the increase of particle size of the nanoemulsion is significantly smaller than that of SPI emulsion. The average particle size in SPI emulsion was most affected by salt concentration and positively correlated with salt concentration, which was significantly higher in both acidic and alkaline conditions ( $\mathrm{p}<0.05)$. TS nanoemulsion remained stable in the range of $0-500 \mathrm{mM}$ salt ion concentration, and its particle size did not change significantly. Therefore, the salt tolerance of SPI emulsion is the worst. When the salt ion concentration of SPI-TS emulsion is more than $400 \mathrm{mM}$, the droplets will aggregate

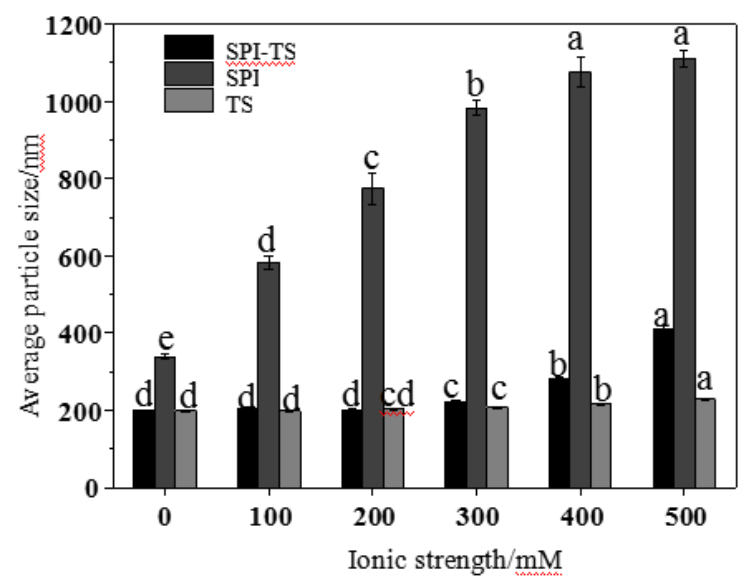

(a)

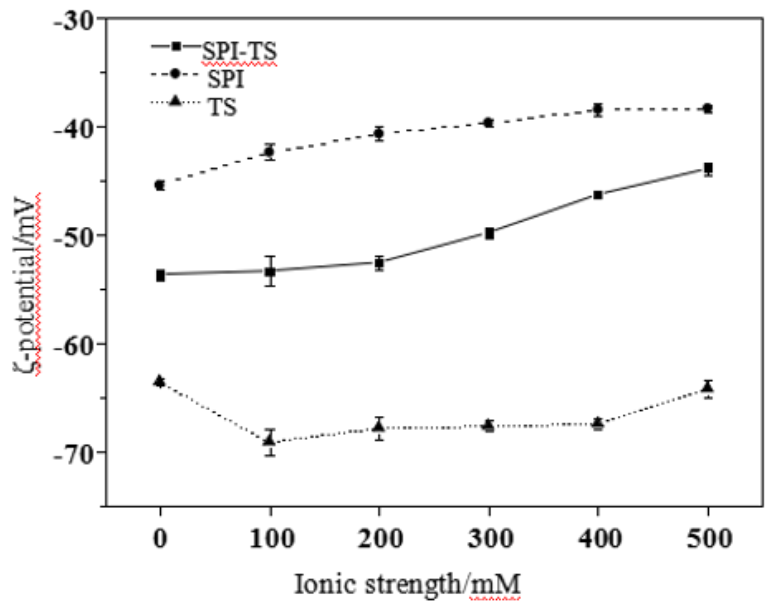

(b)

Fig.3. Effects of different salt ion concentrations on (a) average particle size and (b) $\xi$-potential of camellia oil nanoemulsion

slightly. Figure $3 \mathrm{~b}$ shows the $\zeta$-potential changes of three camellia oil nanoemulsions. The $\zeta$-potential absolute value of SPI-TS nanoemulsion decreases with the increase of salt ion concentration. The $\zeta$-potential absolute value of SPI-TS nanoemulsion decreases gradually after the salt ion concentration reaches 300 $\mathrm{mM}$, which is consistent with the result of Figure $3 \mathrm{a}$. This may be caused by the electrostatic shielding effect [33].

Figure 4 is the confocal laser scanning microscope (CLSM) image of three nanoemulsions under different salt ion concentrations. The CLSM image shows that TS nanoemulsion is extremely resistant to salt ions, and no droplet aggregation is observed in the salt ion concentration range of $0-500 \mathrm{mM}$. In the SPI-TS emulsion, only small droplets aggregated to form large droplets at $500 \mathrm{mM}$. However, when the salt ion concentration of the SPI nanoemulsion is $100 \mathrm{mM}$, the emulsion has already begun to accumulate oil droplets, and as the salt ion concentration increases, the oil droplet aggregation phenomenon becomes more serious.

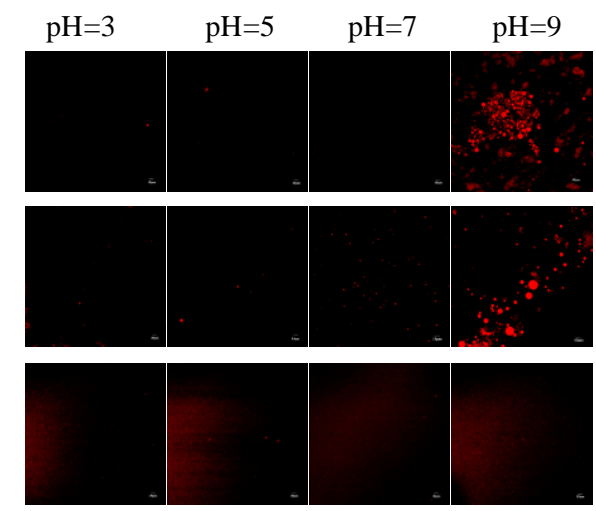

SPI-TS

Fig.4. CLSM diagram of composite nanoemulsion, SPI nanoemulsion and TS nanoemulsion at different salt ion concentrations

\subsubsection{Temperature stability}


Figure 5 is the graph showing the influence of different heating temperatures on the average particle size and $\zeta$ potential of the camellia oil nanoemulsion prepared by three emulsifiers. It can be seen from Figure 5a that after the three nanoemulsions were heated at $30^{\circ} \mathrm{C}-90^{\circ} \mathrm{C}$ for 30 minutes, the average particle size did not change significantly, and the emulsion remained stable. It can be seen from Figure $5 b$ that different heating temperatures have no significant effect on the $\zeta$-potential of SPI-TS emulsion, SPI emulsion and TS emulsion. However, the average particle size of the SPI emulsion was significantly higher than the other two emulsions $(p<0.05)$, and the absolute value of $\zeta$-potential was also significantly lower than the other two emulsions $(\mathrm{p}<0.05)$.

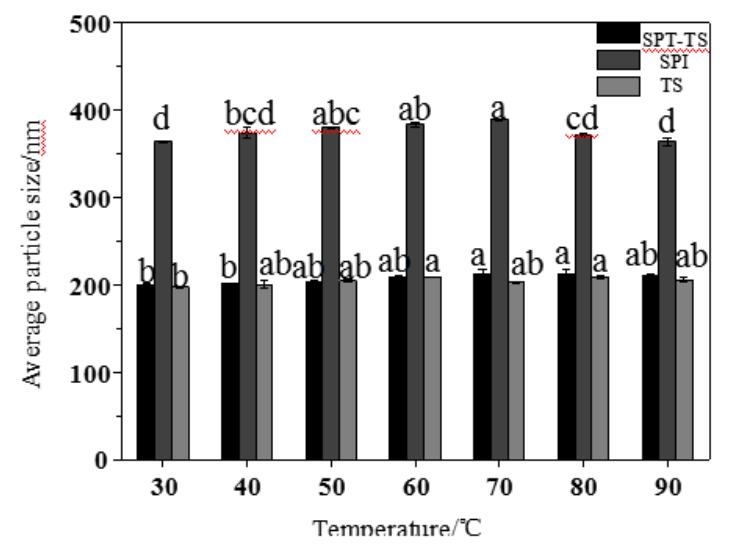

(a)

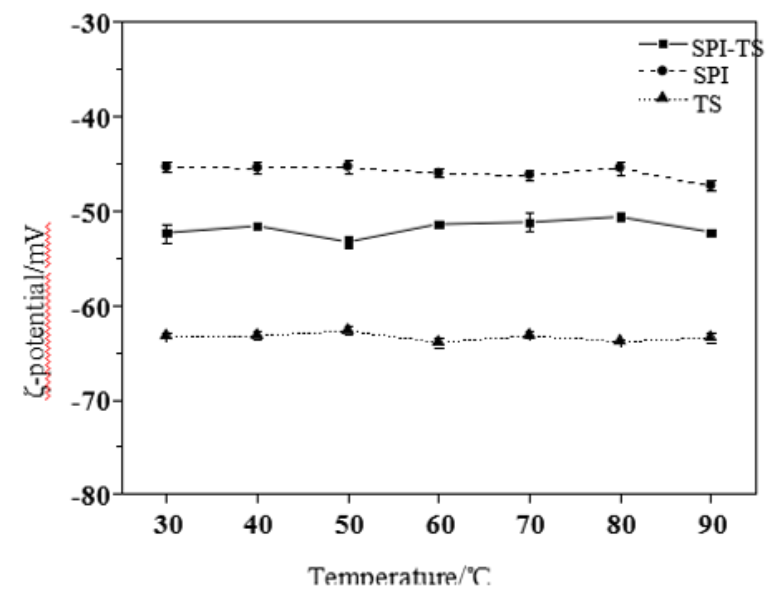

(b)

Fig.5 Effects of different heating temperature on (a) average particle size and (b) $\xi$-potential of camellia oil nanoemulsion

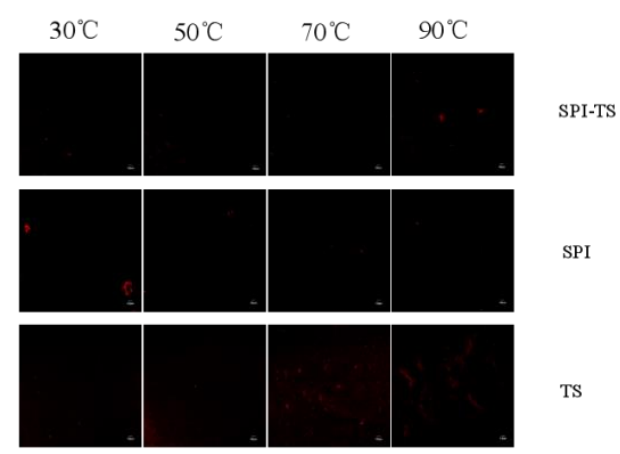

Fig.6. CLSMdiagram of composite nanoemulsion, SPI nanoemulsion and TS nanoemulsion at Different heating temperature

It can be seen from Figure 6 that the three emulsions were heated at different temperatures for 30 minutes, and oil droplet aggregation occurred. From the results in Figure 5 and Figure 6, it can be inferred that the camellia oil nanoemulsion prepared by the three emulsifiers all have good thermal processing tolerance.

\subsubsection{Storage stability}

Figure 7 is the graph showing changes in the average particle size and $\xi$-potential of the three nanoemulsions at storage temperatures of $4^{\circ} \mathrm{C}, 25^{\circ} \mathrm{C}$, and $50^{\circ} \mathrm{C}$ for 28 days. In general, the camellia oil nanoemulsion prepared by SPI-TS composite emulsifier is more stable when stored at $4{ }^{\circ} \mathrm{C}, 25{ }^{\circ} \mathrm{C}$ and $50{ }^{\circ} \mathrm{C}$ for 28 days. It can be seen from Figure 7(a) that at a storage temperature of $4^{\circ} \mathrm{C}$, the average particle size of SPI-TS nanoemulsion remained almost unchanged within 28 days. The particle size of the TS emulsion became slightly larger after storage for 21 days. The average particle size of the emulsion began to increase when the emulsion was stored for 7 days. Through the significance analysis, the average particle size of the three camellia oil nanoemulsions did not change significantly within 28 days, and there was no phenomenon such as emulsion flocculation or fat precipitation. Figure 7(b) shows that the $\xi$-potential of the nanoemulsion prepared by the SPITS composite emulsifier did not change significantly during the 28-day storage period, and the $\xi$-potential of the emulsion remained basically constant. SPI nanoemulsion has a decreasing trend in the absolute value of $\xi$-potential from the 14th day, and the absolute value of $\xi$-potential of TS nanoemulsion drops significantly after storage for 14 days, and the decrease of the absolute value of $\xi$-potential is not conducive to the stability of the emulsion ${ }^{[30,31]}$. 


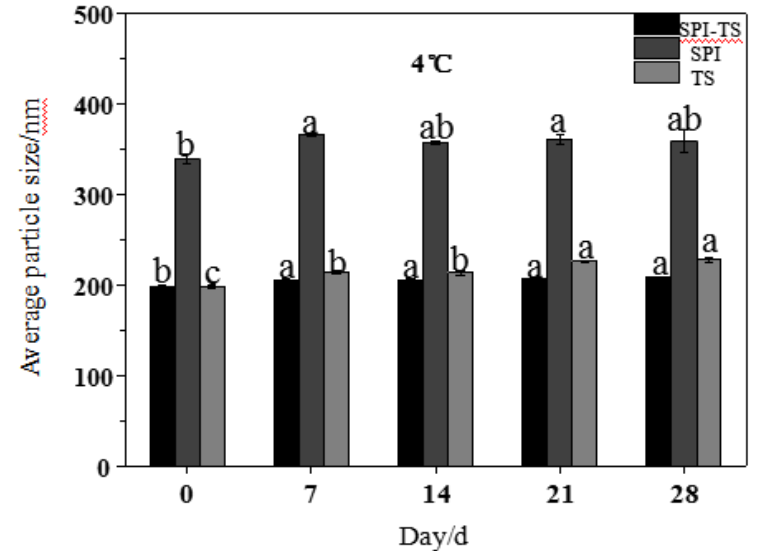

(a)

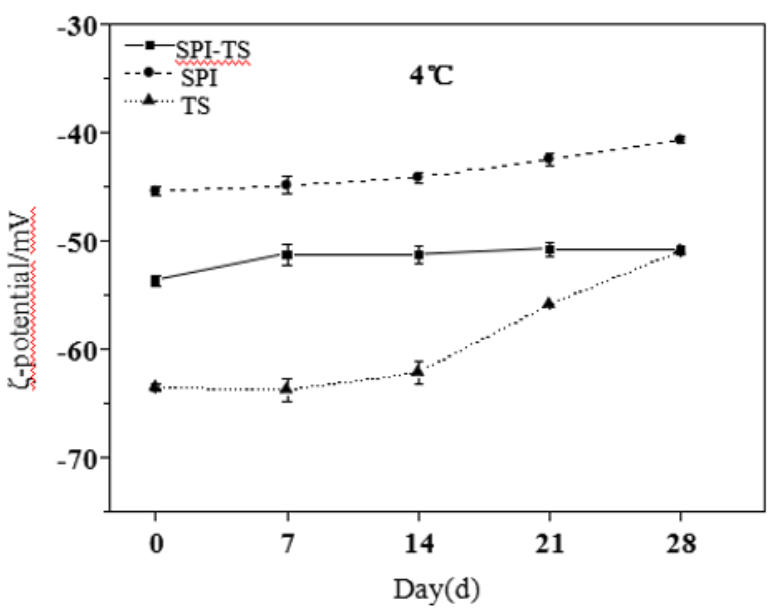

(b)

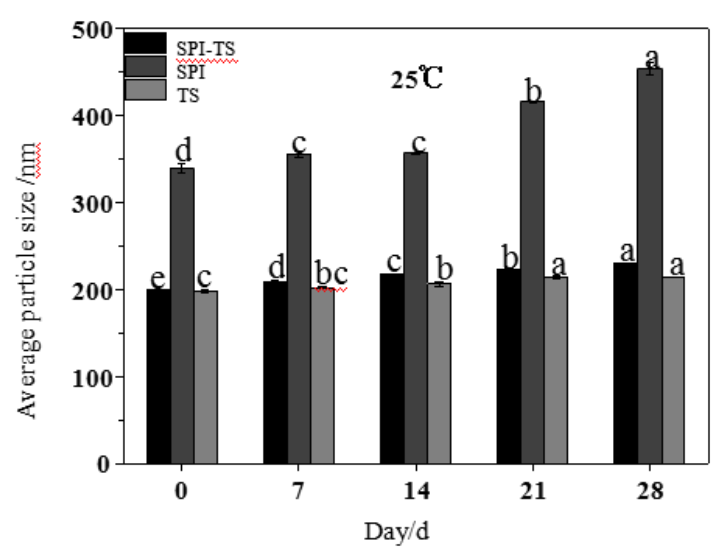

(c)

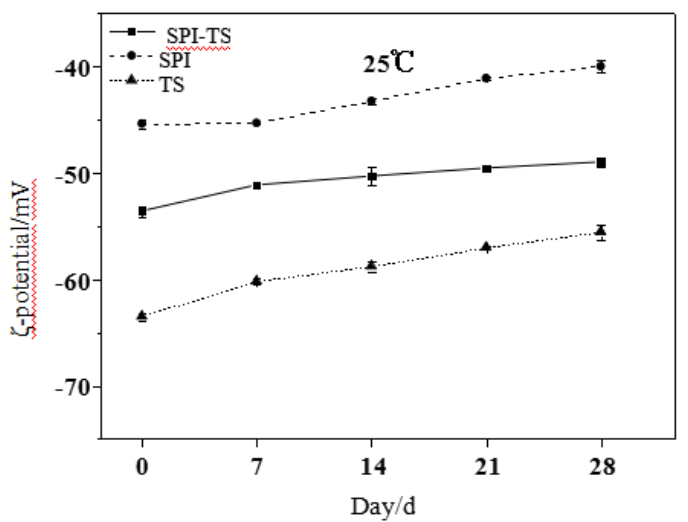

(d)

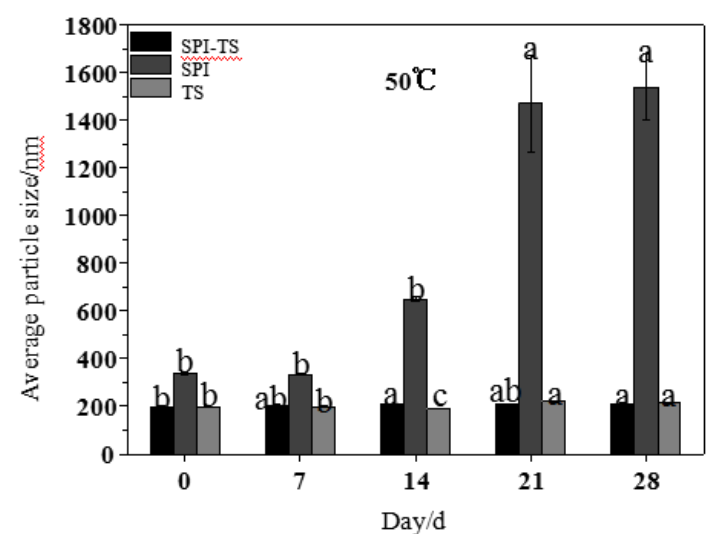

(e)

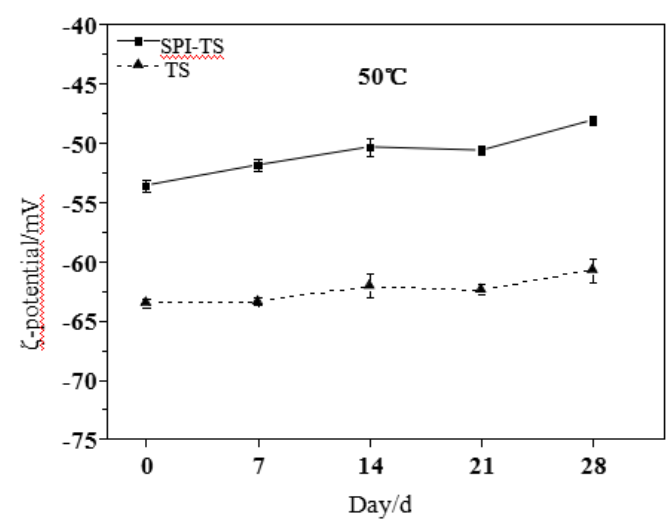

(f)

Fig.7 Effect of storage time on average particle size and $\xi$ potential of camellia oil nanoemulsion at storage temperature $(\mathrm{a}, \mathrm{b}): 4^{\circ} \mathrm{C}$ Average particle size and $\xi$-potential at $4{ }^{\circ} \mathrm{C}$.

(c, d) : Average particle size and $\xi$-potential at $25^{\circ} \mathrm{C}$.

(e, f) : Average particle size and $\xi$-potential at $50^{\circ} \mathrm{C}$.

The three kinds of nanoemulsions were stored at $25^{\circ} \mathrm{C}$ for 28 days. Figure 7(c) of the average particle size and Figure 7(d) of $\xi$-potential show that there is no significant difference in the average particle size of SPITS nanoemulsion and TS nanoemulsion during storage. The average particle size of the SPI nanoemulsion showed an increasing trend, and the average particle size of the emulsion increased significantly after 14 days. Therefore, the SPI nanoemulsion exhibits poor storage stability at $25^{\circ} \mathrm{C}$. According to the results of the $\xi$ - 
potential change, it can be judged that the absolute value of the $\xi$-potential of the three nanoemulsions all show a downward trend during the 28 -day storage period. The $\xi$ potential value of SPI-TS nanoemulsion decreased the least, indicating that SPI-TS nanoemulsion had the best stability when stored at $25^{\circ} \mathrm{C}$ for 28 days. This may be because soy protein isolate and tea saponin can form a very tight interfacial film on the surface of the oil droplets, which increases the electrostatic and spatial repulsion between the droplets, which is more conducive to the stability of the emulsion ${ }^{[28]}$.

The changes in the average particle size of the three nanoemulsions in Figure 7(e) and $\xi$-potential Figure 7(f) are tested at $50{ }^{\circ} \mathrm{C}$. The results showed that the average particle size of the camellia oil nanoemulsion prepared by SPI increased significantly after storage for 14 days $(p<0.05)$ and small oil droplets gathered to form large oil droplets. At this time, the emulsion has condensation, thickening and stratification. Considering that the SPI nanoemulsion has been severely coagulated and stratified, Figure $9 \mathrm{~b}$ does not list its $\xi$-potential change diagram. During the storage period of SPI-TS nanoemulsion and TS nanoemulsion at $50{ }^{\circ} \mathrm{C}$, the absolute value of the $\xi$-potential of both showed a downward trend, but there was no significant difference, indicating that the two have good stability at a high temperature of $50^{\circ} \mathrm{C}$. However, after 60 days of storage at $50^{\circ} \mathrm{C}$, the two emulsions had stratification but did not coagulate and thicken, and the stratification of the emulsion stabilized by TS alone was slightly more obvious than that of the emulsion stabilized by the composite emulsifier. With long-term storage at high temperature due to the increase of Brownian motion, the probability of collision between droplets is greater and the number of times is greater, resulting in flocculation of emulsion droplets ${ }^{[34]}$.

\subsection{In vitro digestion of camellia oil nanoemulsion}

\subsubsection{Change of average particle size during the simulated digestion process}

The type of emulsifier affects the digestion characteristics of the nanoemulsion, and the change of the average particle size of the nanoemulsion during the digestion process will directly affect the bioavailability of the embedded material. In this research, the average particle size of camellia oil nanoemulsion prepared by three different emulsifiers was tested in the four stages of simulated digestion (original emulsion, simulated gastric juice $1 \mathrm{~h}$, simulated intestinal juice $1 \mathrm{~h}$, simulated intestinal juice $2 \mathrm{~h}$ ), as shown in Figure 8.

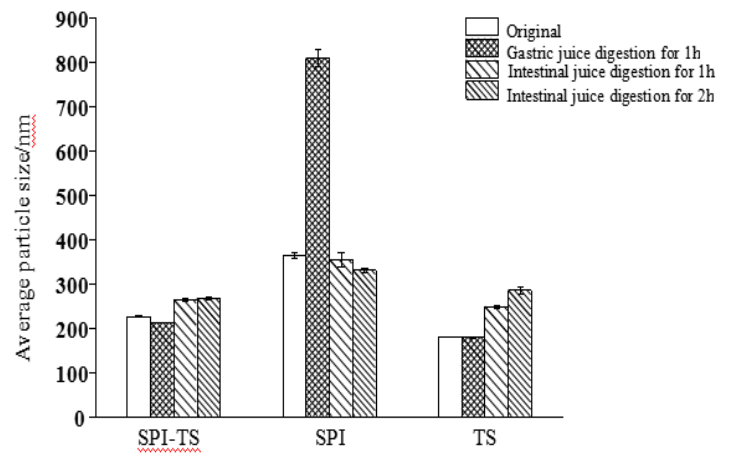

Fig.8. The change of average particle size of camellia oil nanoemulsion during simulated digestion

Figure 8 is the graph showing the average particle size change of three kinds of camellia oil nanoemulsion during simulated digestion.It can be seen from the figure that the digestion characteristics of these three nanoemulsions are obviously different. The average particle size of the camellia oil nanoemulsion prepared by SPI was significantly increased after simulating gastric juice digestion for 1 hour $(\mathrm{p}<0.05)$. This may be due to the large amount of pepsin in the gastric juice, which caused a large amount of protein hydrolysis and weakened the electrostatic repulsion between the droplets, resulting in a sharp increase in the average particle size of the nanoemulsion stabilized by protein emulsifiers $[10,35,36]$. On the other hand, it may be that when the $\mathrm{pH}$ of the gastric juice is near the SPI isoelectric point, it promotes the flocculation of the emulsion. When the gastric juice contains metal ions, the electrostatic repulsion of the emulsion droplets is weakened, and the emulsion polymerizes to increase the particle size [37]. However, SPI-TS and TS nanoemulsions are not affected by protease hydrolysis in gastric juice due to the presence of saponin molecules, and the particle size of the emulsions did not increase significantly. After 1 hour of digestion of simulated intestinal juice, the average particle size of the SPI nanoemulsion decreased. This may be because the large oil droplets of camellia oil were hydrolyzed by pancreatic enzyme and bile saline in the intestinal juice to form small oil droplets after digestion with gastric juice. However, the SPI-TS and TS nanoemulsions showed an increase in particle size during the digestion stage of the simulated intestinal juice. It may be because the tight interface membrane formed by the emulsifier is gradually destroyed during the digestion stage of the intestinal juice, and at the same time it is hydrolyzed by the pancreatic enzyme and bile saline in the intestinal juice, which promotes the release and accumulation of camellia oil droplets.

\subsubsection{Changes in $\xi$-potential during simulated} digestion 


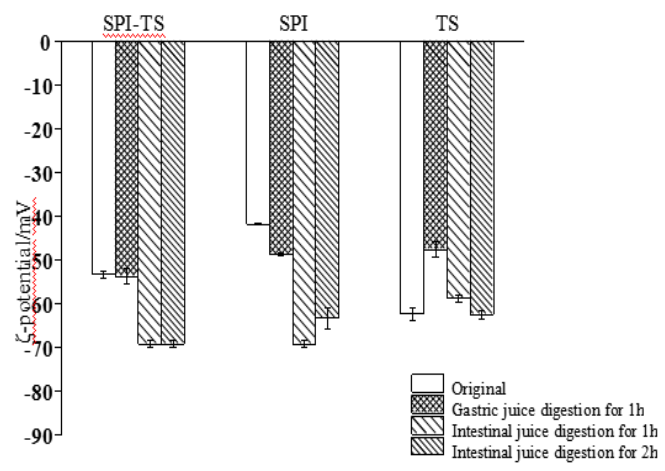

Fig.9. The change of $\xi$-potential of camellia oil nanoemulsion during simulated digestion

The $\zeta$-potential of the emulsion after in vitro digestion can reflect some information about the change of interface composition caused by the substitution of surface active substances [38]. In this research, three different emulsifiers camellia oil nanoemulsion were tested in the four stages of simulated digestion (original emulsion, simulated gastric juice $1 \mathrm{~h}$, simulated intestinal juice $1 \mathrm{~h}$, simulated intestinal juice $2 \mathrm{~h}$ ) changes in $\xi$ potential (Figure 9). SPI-TS and SPI two emulsions increase in absolute value of $\xi$-potential after digestion with gastric juice, and increase in absolute value of $\xi$ potential after digestion with intestinal juice. This may be because camellia oil is hydrolyzed by pancreatic lipase and releases free anions with anions. Fatty acids and monoglycerides increase the net charge of the digestive system. On the other hand, it may be because the $\mathrm{pH}$ of the digestive system of intestinal juice is around 7.0, and negatively charged lipids, bile salts and other substances are easily adsorbed to oil droplets or micelles. On the surface, the nanoemulsion has a large amount of negative charge after digestion of intestinal juice, so that the absolute value of the $\xi$-potential of the whole system is greater than that of the original emulsion ${ }^{[39]}$. However, the absolute value of the $\xi$ potential of TS nanoemulsion did not increase significantly after digestion in the intestinal juice, which may be because the saponin emulsifier was relatively stable during the digestion process, and the amount of free fatty acids with negatively charged oil was hydrolyzed into less. As confirmed by Figure 12, the TS nanoemulsion shows a lower free fatty acid release rate.

\subsubsection{Release of free fatty acids (FFAs)}

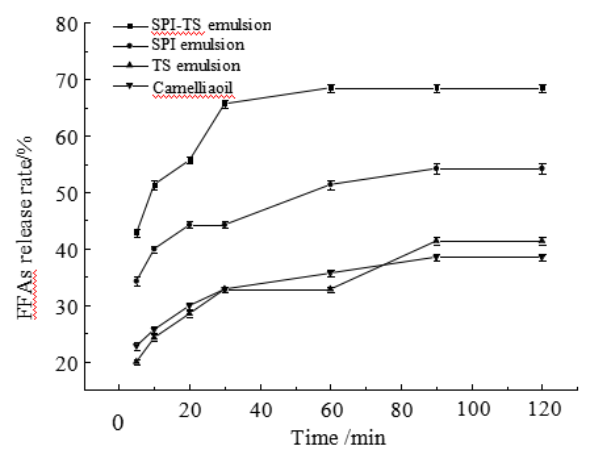

Fig.10. Comparison of free fatty acid release rate of camellia oil nanoemulsion and control group (camellia oil) during simulated intestinal digestion

The FFAs release rate of the three kinds of camellia oil nanoemulsions during the digestion process of simulated intestinal juice was compared with that of the control group (camellia oil) to judge the degree and rate of hydrolysis of camellia oil during the digestion process. The results of the research are shown in Figure 10. The FFAs release rate of the SPI-TS camellia oil nanoemulsion can reach $68.46 \%$, which is significantly higher than the control group without emulsifier $(38.51 \%)$. The order of free fatty acid release rate is SPITS $(68.46 \%)>$ SPI $(54.20 \%)>$ TS $(41.36 \%)>$ camellia oil $(38.51 \%)$. Although the fatty acid release rates of nanoemulsions prepared by the three emulsifiers are significantly different, they are all higher than the control group, which is consistent with the results of Karthik P et al. ${ }^{[40]}$. The digestibility of lipids largely depends on the type of emulsifier used in the preparation process. The hydrolysis rate of SPI-TS nanoemulsion oil is the fastest during $10-30 \mathrm{~min}$. This result indicates that the nanoemulsion prepared by the composite emulsifier can significantly improve the hydrolysis rate and digestibility of camellia oil.

\section{Conclusion}

Based on the poor stability of the nanoemulsion carrier system in the commercial application environment, the $\mathrm{pH}$, salt ion concentration, heating temperature and storage stability of the camellia oil nanoemulsion prepared by the SPI-TS composite emulsifier were studied, and compared with SPI and TS alone. The prepared emulsions were compared and the results showed that SPI-TS is a natural compound emulsifier, which greatly improves the environmental stability of the emulsion compared with SPI emulsifier. The prepared nanoemulsion can exist stably in the $\mathrm{pH}$ range of 5-9, while SPI nanoemulsion is extremely unstable in acidic environment. SPI-TS nanoemulsion can withstand the influence of salt ions $(\mathrm{NaCl})$ in a certain concentration range $(0-300 \mathrm{mM})$, but it does not tolerate high salt environment $(500 \mathrm{mM})$. After heating at $30^{\circ} \mathrm{C}$ $90{ }^{\circ} \mathrm{C}$ for $30 \mathrm{~min}$, the average particle size and $\xi$ potential of the three emulsions remained stable, and no 
droplet aggregation was observed in the three CLSM images, showing good thermal stability. After being stored at 4,25 , and $50^{\circ} \mathrm{C}$ for four weeks, the average particle size and $\xi$-potential of the SPI-TS and TS nanoemulsions did not change significantly, and the storage stability of the two was good. The results of in vitro simulated digestion showed that the digestibility of emulsions prepared with different emulsifiers is significantly different. SPI-TS nanoemulsion can significantly improve the digestibility of camellia oil. The FFAs release rate of the three nanoemulsions is higher than that of the control group. The emulsion delivery system can effectively improve the digestibility of camellia oil.

This work was supported by Central Public-interest Scientific Institution Basal Research Fund for Chinese Academy of Tropical Agricultural Sciences(NO. 1630092019001, 1630092020011) and the Major scientific and technological projects in Hainan Province (ZDKJ2017004).

\section{References}

1. M. Caser, D. Marinoni, V. Scariot, Genome, 53: 384-399, (2010)

2. X. Fang, X. Fei, H. Sun, Y. Jin, Eur. J. Lipid Sci. Technol. 118: 244-251. (2016)

3. X. Cheng, T. Yang, Y. Wang, B. Zhou, L. Yan, L. Teng, et al.. Arab. J. Chem.11(6): 815-826.(2018)

4. J. Yang, B. Peng, M. Wang. Food Hydrocolloids 87: 644-652. (2019)

5. N. Guo, T. Tong, N. Ren, Y. Tu, B. Li. Phytochemistry, 149: 42-55.(2018)

6. X. Wang, Q. Zeng, V. Verardo, M. Contreras. Food Chem. 233: 302-310. (2017)

7. Y. Ye, H. Xing, Y. Li. Int. J. Nanomed. 9: 44754484. (2014)

8. H. Wu, C. Li, Z. Li, R. Liu, A. Zhang, Z. Xiao L. Ma, J. Li, S. Deng. Fuel Process Technol. 174: 8894. (2018)

9. S. Zhang, L. Zheng, B. Ai, X. Zheng, Y. Yang, Y. Pan, Z. Sheng. J. Food Sci. 40:124-130.(2019) (in Chinese)

10. Z. Hong, W. Wu, L. Li, Y. Li, X. Xie. J. Food Sci. 40:1-7.(2019) (in Chinese)

11. M. Shah, M. Imran, S. Ullah. William Andrew:111137.(2017)

12. Y. Guan, J. Wu, Q. Zhong. Food Chem. 194: 787796. (2016)

13. J. Yi, T. Lam, W. Yokoyama. L. Cheng, F. Zhong. J. Agric. Food Chem. 62: 1096-1104. (2014)

14. X. Li, K. Li, Y. Shen, F. Niu, Y. Fu. Colloid Surface. A 504: 442-448. (2016)

15. Y. Jo, Y. Kwon. Food Sci Biotechnol 23:107113.(2014)

16. Y. Wang. Shanxi University of Science and Technology, (2017) (in Chinese)
17. G. Aken.. Soft Matter 6:826-834.(2010)

18. J. Ferruam, R. Singh. J. Food Sci. 75: 151-162. (2010)

19. F. Kong,R. Singh. J. Food Sci. 73:67-80.(2008)

20. X. Luo , Y. Zhou, L. Bai, F. Liu, R. Zhang, Z. Zhang, B. Zheng, Y. Deng, D. McClements. Food Res. Int. 96: 103-112. (2017)

21. Y. Zhang, F. Jiang, Q. Liu, J. Han, P. He. Food Research And Development 38:1-5. (2017) (in Chinese)

22. Y. Zhu, S. Zhao, R. An, X. Zhang, Z. Wang, L. Jiang. J. Agric. Machinery 50 :330-335.(2019) (in Chinese)

23. Q. Li, Y. Tao, Z. Wan, J. Qi, X. Yang. J. Food Sci. 40:9-15. (2019) (in Chinese)

24. D. Mcclements, L. Bai, C. Chung. Annu. Rev. Food Sci. T. 8: 205-236.(2017)

25. Z. Zhu, Y. Wen, J. Yi, Y. Cao, F. Liu, D. McClements. J. Colloid Interface Sci. 536:80-87. (2019)

26. T. Wooster, L. Day, M. Xu, M. Golding, S. Oiseth, J. Keogh, P. Clifton. Food Hydrocolloid. 36:102114.(2014)

27. Y. Li, D. McClements. J. Agric. Food Chem. 58:8085-8092. (2010)

28. X. Xu, Q. Sun, D. Mcclements. Food Hydrocolloids, 89:396-405. (2019)

29. L. Jiang, Y. Qi, C.Ma, B. Liu. J. Agric. Machinery 49:387-395.(2018)

30. S. Böttcher, S. Drusch. Adv. Colloid Interface Sci. 243 :105 - 111. (2017)

31. Y. Yang, M. Leser, A. Sher, D. Mcclements. Food Hydrocolloids 30:589-596.(2013)

32. Y. Li , M. Li, Y. Qi, L. Zheng. Food Hydrocolloids, 100.(2020)

33. D. Mcclements. CRC press.(2016)

34. R. Liang, S. Xu, C. Shoemaker, Y. Li, F. Zhong, Q. Huang. J. Agric. Food Chem. 60:7548-7555. (2012)

35. N. Malaki, A. Wright, M. Corredig. Food Funct. 1:141-148. (2010)

36. J. Yi , Y. Li , F. Zhong, W. Yokoyama. Food Hydrocolloid. 35: 19-27.(2014)

37. N. Malaki, A. Wright , M. Corredig. Colloids Surf. B 83 : 321-330.(2011)

38. D. Mcclements, Y. Li. Food Funct. 1:32-59. (2010)

39. Z. Wang, X. Zhang, J. Che, Y. Li, O. Olga, L. Jiang. Journal of Agricultural Machinery 49:381-386. (2018)

40. P. Karthik, C. Anandharamakrishnan. J. Food Eng. 187:92-105. (2016) 